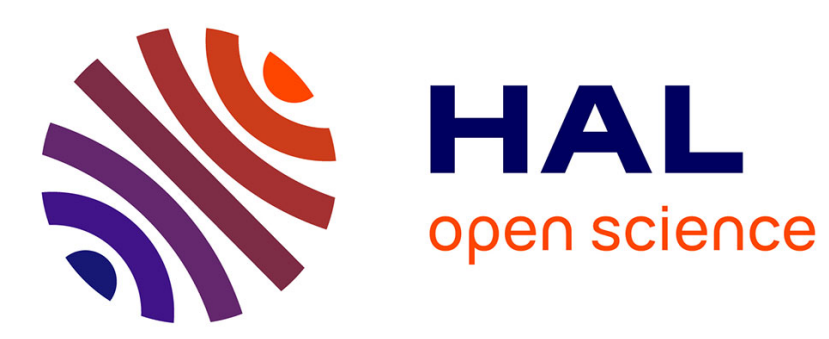

\title{
Oxydation catalytique du silicium : l'obtention de couches d'oxydes uniformes
}

Daniel Roy, B. Lamontagne

\section{To cite this version:}

Daniel Roy, B. Lamontagne. Oxydation catalytique du silicium : l'obtention de couches d'oxydes uniformes. Journal de Physique IV Proceedings, 1997, 07 (C6), pp.C6-127-C6-135. 10.1051/jp4:1997611 . jpa-00255710

\section{HAL Id: jpa-00255710 https://hal.science/jpa-00255710}

Submitted on 1 Jan 1997

HAL is a multi-disciplinary open access archive for the deposit and dissemination of scientific research documents, whether they are published or not. The documents may come from teaching and research institutions in France or abroad, or from public or private research centers.
L'archive ouverte pluridisciplinaire HAL, est destinée au dépôt et à la diffusion de documents scientifiques de niveau recherche, publiés ou non, émanant des établissements d'enseignement et de recherche français ou étrangers, des laboratoires publics ou privés. 


\title{
Oxydation catalytique du silicium : l'obtention de couches d'oxydes uniformes
}

\author{
D. Roy et B. Lamontagne( $\left.{ }^{1}\right)$ \\ Centre de Recherche sur les Propriétés des Interfaces et la Catalyse (CERPIG) et Laboratoire de \\ Physique Atomique et Moléculaire (LPAM), Département de Physique, Pavillon Vachon, Université \\ Labal, Québec, G1K 7P4, Canada
}

\begin{abstract}
Résumé : L'oxydation catalytique du silicium (111) à l'aide des métaux alcalins, particulièrement le potassium, est étudiée dans notre groupe depuis plusieurs années, à l'aide de diverses techniques. Il est bien connu qu'à température ambiante, on ne peut déposer qu'une monocouche de potassium et qu'il n'en résultera qu'une faible quantité d'oxyde sous forme d'une mince couche uniforme. Le défi est de déterminer dans quelles conditions on peut arriver à obtenir des couches plus épaisses et toujours uniformes. Ainsi, sur un échantillon de $\mathrm{Si}(111)$ refroidi à environ $-125^{\circ} \mathrm{C}$, il est possible de déposer une plus grande quantité de potassium. Toutefois, tel que confirmé par SEM, micro-AES et AFM, le mécanisme d'adsorption de potassium à cette température est caractérisé par une croissance en îlots du type Stranski-Krastanov. Après exposition à l'oxygène, la désorption subséquente du potassium à température modérée $\left(700^{\circ} \mathrm{C}\right)$ donne des îlots de $\mathrm{SiO}_{2}$ entourés d'une mince couche uniforme de $\mathrm{SiO}_{2}$. Cependant nos travaux ont montré que l'on peut obtenir une couche uniforme de $\mathrm{SiO}_{2}$ si la couverture de potassium est exposée à une basse pression d'oxygène $\left(10^{-8} \mathrm{mbar}\right)$. On peut arriver au même résultat si l'adsorption de potassium s'effectue simultanément avec l'exposition à l'oxygène à température ambiante. L'élimination finale du potassium est confirmée par des mesures SIMS,
\end{abstract}

\begin{abstract}
The catalytic oxydation of silicon (111) by alkali metals, mainly potassium, has been studied in our group for many years, by mean of various techniques. It is well known that, at room temperature, only one monolayer of potassium can be deposited, resulting in a thin uniform oxide layer. The challenge is to determine which conditions can yield thicker, still uniform, oxide layers. For instance, on a sample of $\mathrm{Si}(111)$ cooled at about $-125^{\circ} \mathrm{C}$, more potassium can be deposited. However, as confirmed by SEM, micro-AES and AFM, the adsorption of potassium at this temperature is characterized by the Stranski-Krastanov growth mode in islands. After exposition to oxygen, the subsequent desorption of potassium at moderate temperature $\left(700^{\circ} \mathrm{C}\right)$ yields $\mathrm{SiO}_{2}$ islands, surrounded by a thin uniform layer of $\mathrm{SiO}_{2}$. Our works have shown that it is possible to obtain a uniform layer of $\mathrm{SiO}_{2}$ if the potassium overlayer is exposed to low oxygen pressure $\left(10^{-8} \mathrm{mbar}\right)$. Similar results can be obtained if potassium deposition is done simultaneously with oxygen exposition at room temperature. The final elimination of potassium is confirmed by SIMS measurements.
\end{abstract}

\section{INTRODUCTION}

L'étude de l'oxydation des métaux alcalins conserve un grand intérêt car elle est reliée à plusieurs sujets de recherche importants, comme la catalyse hétérogène [1], les matériaux à faibles travaux de sortie [2] et particulièrement l'oxydation catalytique des semiconducteurs [3-8]. Dans ce dernier cas, c'est la quête de l'intégration des composants de la microélectronique poussée à une échelle de plus en plus grande qui amène à rechercher de nouveaux procédés pour l'obtention de couches d'oxydes de plus en plus fines (moins de $100 \AA$ ). Comme le procédé classique d'oxydation à haute température est susceptible d'entraîner des effets secondaires indésirables comme la diffusion des dopants ou de certaines impuretés, un procédé efficace à plus basse température est donc souhaitable.

Il a été démontré [3-8] que les métaux alcalins permettaient d'augmenter considérablement le taux d'oxydation du silicium et qu'ils pouvaient ensuite être éliminés par un chauffage à température modérée (environ $650^{\circ} \mathrm{C}$ ). Il existe une littérature abondante sur les mécanismes qui interviennent dans ce procédé et quelques points sont sujets à controverse. Il faut distinguer deux principaux cas : celui de la monocouche, qui s'obtient généralement à température ambiante, et celui des multicouches, qui exige normalement un dépôt sur un échantillon refroidi, suivi de l'exposition à l'oxygène (adsorption séquentielle). Si le premier cas donne une mince couche de $\mathrm{SiO}_{2}$ uniforme sans difficulté particulière, il n'en est pas de mềme du second cas. C'est pourquoi ces dernières années nous avons travaillé sur ce sujet pour arriver à mieux comprendre les mécanismes en jeu et déterminer certaines procédures permettant d'obtenir une couche plus

$\left({ }^{1}\right)$ Maintenant à l'Institut des Sciences des Microstructures, Conseil National de Recherches du Canada, Édifice M-50, Chemin Montréal, Ottawa (Ontario) K1A 0R6, Canada 
épaisse d'un oxyde toujours uniforme et utilisable technologiquement. Le cas de l'adsorption simultanée à température ambiante a également fait l'objet de nos travaux.

Le présent texte se propose donc de présenter une synthèse de nos travaux sur l'obtention de multicouches d'oxydes uniformes de $\mathrm{SiO}_{2}$ sous l'action catalytique du potassium, dans le cas de $\mathrm{Si}(111)$.

\section{CONDITIONS EXPÉRIMENTALES}

Les travaux présentés font appel à des mesures réalisées par différentes techniques, soit principalement la spectroscopie de photoélectrons induits par rayons X (XPS), la microscopie électronique à balayage (SEM), la spectroscopie Auger (AES) et la spectrométrie de masse des ions secondaires (SIMS). Ces mesures ont été réalisées sur l'appareil VG ESCALAB MarkII du Laboratoire d'analyse de surfaces (LAS) du CERPIC, qui comprend trois chambres distinctes, soit les chambres d'analyse en énergie et d'analyse en masse, séparées par la chambre de préparation.

Nos échantillons de $\mathrm{Si}(111)$ sont dopés n $(5-10 \Omega \cdot \mathrm{cm})$. Le nettoyage s'effectue dans la chambre de préparation par bombardement électronique, à environ $1100^{\circ} \mathrm{C}$ pendant $20 \mathrm{~s}$. L'état de propreté est contrôlé par XPS. Le dépôt de potassium est effectué au moyen de sources SAES. À température ambiante, la saturation s'obtient pour une couverture de une monocouche (MC) de potassium. En refroidissant l'échantillon à environ $-125^{\circ} \mathrm{C}$, il est possible d'adsorber une plus grande quantité de potassium : c'est le cas des multicouches. L'exposition à l'oxygène est normalement réalisée dans la chambre de préparation, à basse pression ( $\left.1 \times 10^{-8} \mathrm{mbar}\right)$ ou à haute pression $\left(5 \times 10^{-6} \mathrm{mbar}\right)$. L'adsorption du potassium et l'exposition à l'oxygène peuvent être réalisées de façon séquentielle ou simultanée; les deux cas seront présentés.

\section{LES DIVERS TYPES D'ADSORPTION}

\subsection{Adsorption séquentielle}

Dans le cas de l'adsorption du potassium en multicouches sur un échantillon refroidi à $-125^{\circ} \mathrm{C}$, on constate par microscopie électronique (fig. 1a) la formation d'ilôts importants superposés à un fond uniforme, tout à fait caractéristique du mode de croissance Stranski-Krastanov. Trois spectres Auger à haute résolution spatiale réalisés sur un îlot et sur le fond respectivement (fig. 1b) témoignent de la forte concentration de potassium présente dans les îlots, alors que le fond laisse entrevoir le silicium sous-jacent. L'épaisseur du fond est de 2 à 3 MC. Un spectre obtenu pour une monocouche (température ambiante) est également présenté pour fin de comparaison.

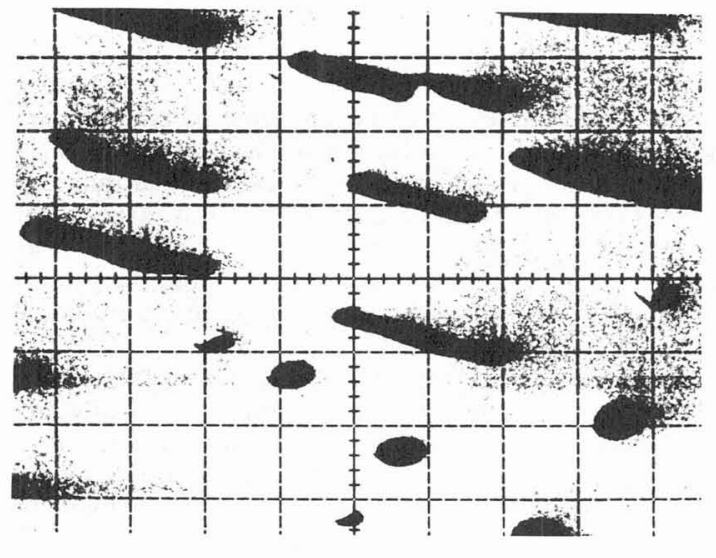

a)

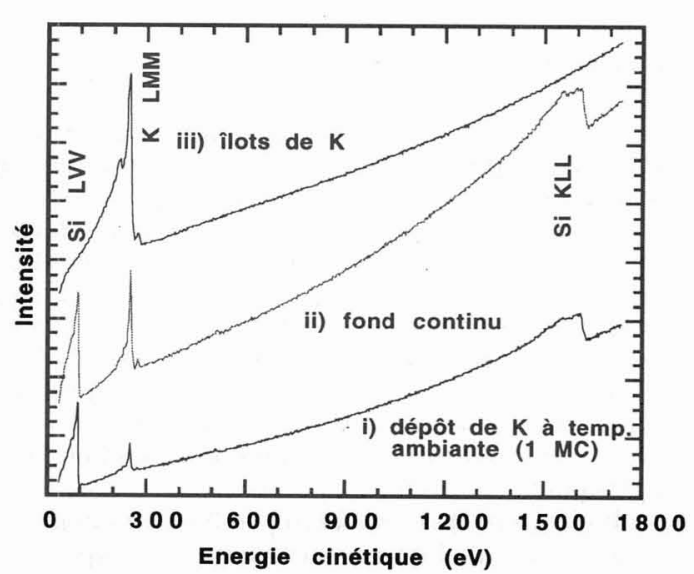

b)

Figure 1 : (a) Image SEM obtenue avec des électrons de $10 \mathrm{keV}$ montrant les îlots de potassium dans le cas des multicouches (chaque carré de la grille a une longueur horizontale de $12 \mu \mathrm{m}$ ). (b) Spectres Auger à très haute résolution spatiale donnant la composition des îlots, du fond entourant les îlots ainsi que pour une monocouche (température ambiante). 
Si un tel échantillon est exposé à $5000 \mathrm{~L}$ d'oxygène $\left(1\right.$ langmuir $=10^{-6}$ Torr. $\left.\mathrm{s}\right)$ à pression élevée $(5 \mathrm{x}$ $10^{-6} \mathrm{mbar}$ ) et que le potassium est subséquemment désorbé par chauffage à $700^{\circ} \mathrm{C}$, on obtient alors des îlots de $\mathrm{SiO}_{2}$ entourés d'une fine couche uniforme du même oxyde. Ces résultats sont présentés à la figure 2 et sont commentés plus en détails dans un article antérieur [9].

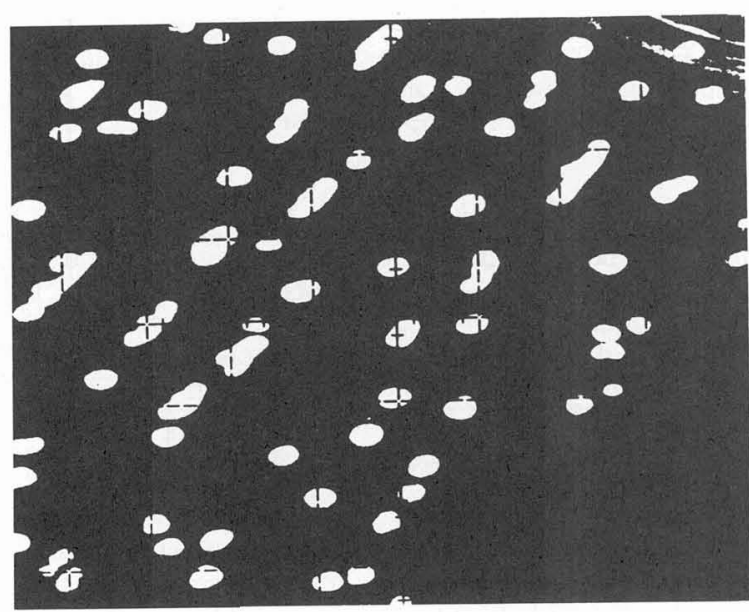

a)

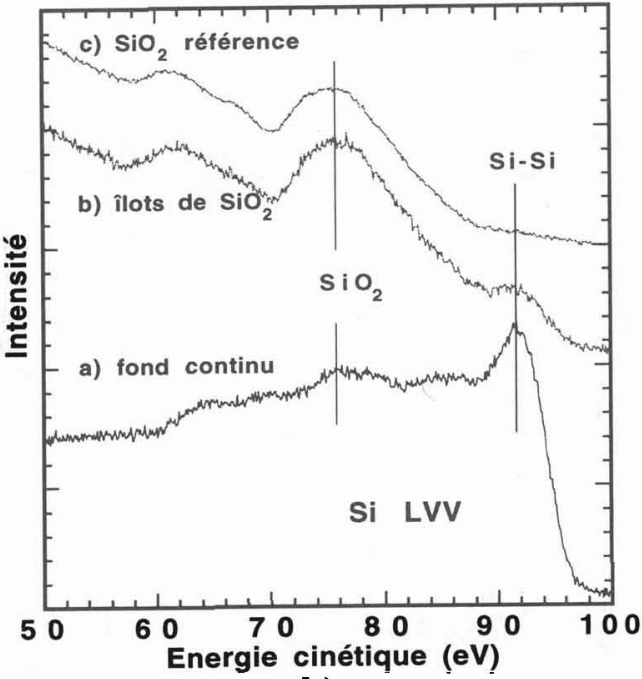

b)

Figure 2 : (a) Image SEM obtenue avec des électrons de $10 \mathrm{keV}$ montrant les îlots de $\mathrm{SiO}_{2}$ après exposition à $5000 \mathrm{~L}$ d'oxygène à pression élevée $\left(5 \times 10^{-6} \mathrm{mbar}\right.$ ) et désorption du potassium à $700^{\circ} \mathrm{C}$ (chaque carré de la grille a une longueur horizontale de $25 \mu \mathrm{m}$ ). (b) Spectres Auger à très haute résolution spatiale mesurés sur les îlots et sur le fond [9].

Pour en arriver à comprendre le processus de formation de ces îlots d'oxyde et si possible empêcher leur formation, nous avons étudié les différentes étapes de formation des oxydes au cours de l'exposition à l'oxygène au moyen de la technique XPS. Ainsi pour un échantillon de $\mathrm{Si}(111)$ gardé à $-125^{\circ} \mathrm{C}$ pendant le dépôt de potassium et l'exposition à l'oxygène, des spectres XPS ont été pris à différents intervalles pendant une longue exposition de $5000 \mathrm{~L}$ afin de suivre l'évolution des pics caractéristiques du silicium (Si $2 p$, avec l'apport des oxydes s'il.y a lieu), du potassium $(\mathrm{K} 2 p)$ et de l'oxygène $(\mathrm{O} 1 s)$. Les graphiques présentés à la figure 3 montrent ces évolutions à partir du moment où le dépôt de potassium a été effectué, suivi de l'exposition graduelle à l'oxygène et, enfin, du chauffage de l'échantillon, d'abord à la température ambiante (TA), puis à $300^{\circ} \mathrm{C}$ et finalement à $700^{\circ} \mathrm{C}$ pour la désorption du potassium. Les graphiques comprennent les intensités obtenues lorsque l'exposition à l'oxygène s'effectue à faible pression (courbes a), à haute pression pour en arriver directement à une exposition élevée de $5000 \mathrm{~L}$ (courbes b) et les intensités dans le cas de la monocouche de potassium sans ou avec refroidissement (courbes c et d).

L'observation la plus significative qui ressort de ce graphique est l'augmentation de l'intensité du pic de potassium dès le début de l'exposition, intensité nettement plus grande dans le cas de l'exposition à faible pression (courbe a) que dans le cas de l'exposition à haute pression (courbe b); le premier cas correspond à la formation d'importants oxydes $\mathrm{K}_{x} \mathrm{O}_{y}$ tout au long du processus. En même temps l'intensité du silicium s'estompe rapidement, alors qu'elle demeure élevée dans le cas $b$, suggérant par là que les îlots s'étalent graduellement sur la surface au cours de l'exposition à faible pression. Pour arriver à comprendre ce qui se passe, il faut s'arrêter aux spectres de $0.1 s$ présentés à la figf́re 4 pour les différentes étapes de l'exposition jusqu'au recuit final. Dès que l'exposition atteint $5 \mathrm{~L}$ (courbe d), on observe la formation de plusieurs oxydes en succession $\left(\mathrm{K}_{2} \mathrm{O}, \mathrm{K}_{2} \mathrm{O}_{2}, \mathrm{KO}_{2}\right)$. La présence de ces divers oxydes avait déjà été mise en évidence dans certains travaux antếrieurs [4, 7, 11-13]. De plus nous notons l'apparition d'une nouvelle composante, à exposition élevée, que nous attribuons à $\mathrm{K}_{2} \mathrm{O}_{3}$. Ce type d'oxyde a été proposé par Su et al. [12] dans le cas du césium. Finalement, lorsque la température de l'échantillon est augmentée, on observe d'abord l'ap- 

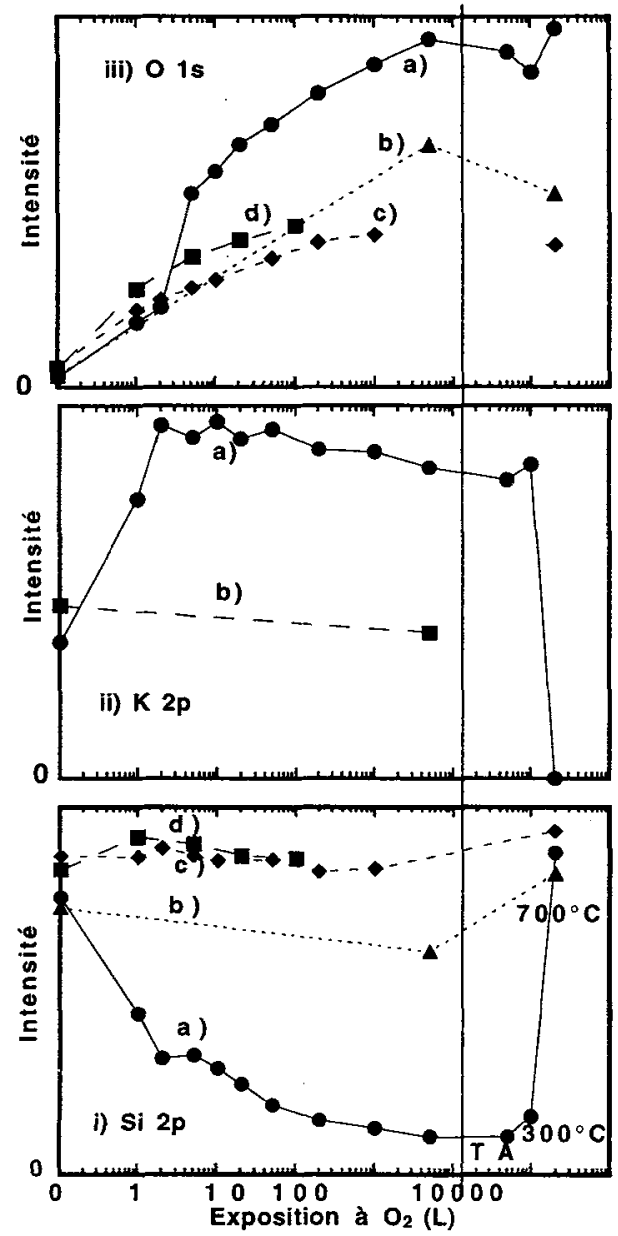

Figure 3 : Intensités des pics $\mathrm{Si} 2 p, \mathrm{~K} 2 p$ et $\mathrm{O} 1 s$ en fonction de l'exposition à l'oxygène et du traitement thermique (côté droit de la ligne verticale) pour différentes combinaisons de procédés d'oxydation et de recouvrement de potassium pour le système $\mathrm{O} / \mathrm{K} / \mathrm{Si}$ : (a) multicouches de $\mathrm{K}$ exposées à une faible pression d'oxygène, (b) multicouches de $\mathrm{K}$ exposées à une pression élevée d'oxygène et (c) monocouche de $\mathrm{K}$.

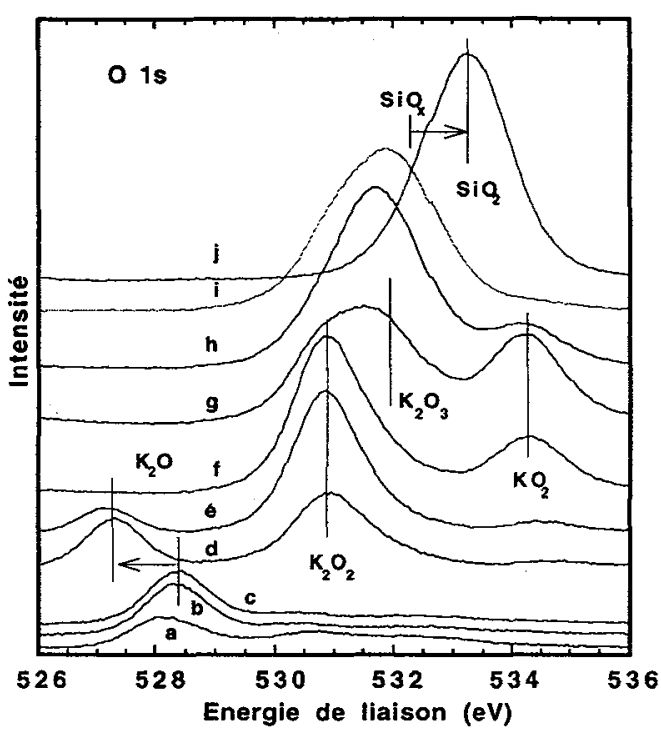

Figure 4 : Spectres XPS de $\mathrm{O} 1 s$ pour des multicouches de $\mathrm{K}$ déposées sur $\mathrm{Si}(111)$ refroidi à $150 \mathrm{~K}$, et soumises à différentes expositions d'oxygène à faible pression : (a) $1 \mathrm{~L}$. (b) $2 \mathrm{~L}$, (c) $3 \mathrm{~L}$, (d) $5 \mathrm{~L}$, (e) $20 \mathrm{~L}$, (f) $200 \mathrm{~L}$, (g) $5000 \mathrm{~L}$, (h) température ambiante, (i) $\sim 300^{\circ} \mathrm{C}$ et $(\mathrm{j})$ après désorption de $\mathrm{K}\left(\sim 700^{\circ} \mathrm{C}\right)$.

parition des sous-oxydes $\mathrm{SiO}_{x}$, puis, après désorption du potassium, le pic caractéristique de $\mathrm{SiO}_{2}$. L'épaisseur de la couche d'oxyde ainsi obtenue est évaluée à 10-20 $\AA$.

$\mathrm{La}$ figure 5 montre l'intensité relative des pics correspondants à ces divers oxydes en fonction de l'exposition à l'oxygène et illustre bien la séquence qui les caractérise en relation avec la durée de l'exposition et indique que l'on a bel et bien un profil de distribution en profondeur propre à chacun de ces oxydes. Des mesures de photoémission XPS résolues angulairement ont permis de confirmer cette relation [10]. Ainsi, pour une faible exposition de $5 \mathrm{~L}$, il est très clair que l'oxyde $\mathrm{K}_{2} \mathrm{O}_{2}$ se superpose à $\mathrm{K}_{2} \mathrm{O}$. De même à exposition élevée ( $5000 \mathrm{~L}$ ), ces mesures mettent en évidence que l'oxyde qui contient le plus grand nombre d'atomes d'oxygène, $\mathrm{K}_{2} \mathrm{O}_{3}$, vient bien se superposer à $\mathrm{K}_{2} \mathrm{O}_{2}$ et $\mathrm{KO}_{2}$. 
Comme le montre la figure 6, on peut suivre par SEM l'évolution des îlots au cours d'une exposition lente à l'oxygène, à basse pression $\left(1 \times 10^{-8} \mathrm{mbar}\right)$. La première image montre les îlots de potassium avant le début de l'exposition, alors que la pression est encore à $4 \times 10^{-10} \mathrm{mbar}$; l'image suivante est obtenue après une exposition de $0.2 \mathrm{~L}$. Dès le début, un important étalement des îlots se produit et ce processus évolue lentement au cours de l'exposition; après la désorption du potassium, on obtient finalement une couche d'oxyde uniforme. Par contre, dans le cas d'une exposition à pression élevée $\left(5 \times 10^{-6} \mathrm{mbar}\right)$, on observe un certain étalement des îlots au début, mais le processus bloque rapidement et les îlots restent figés jusqu'à la fin. On retrouve finalement des îlots très nets de $\mathrm{SiO}_{2}$ d'une hauteur d'environ $20 \mathrm{~nm}$ et de dimensions latérales de l'ordre de $10 \mu \mathrm{m}$, tel que confirmé par SEM et par microscopie à force atomique (AFM) [9].

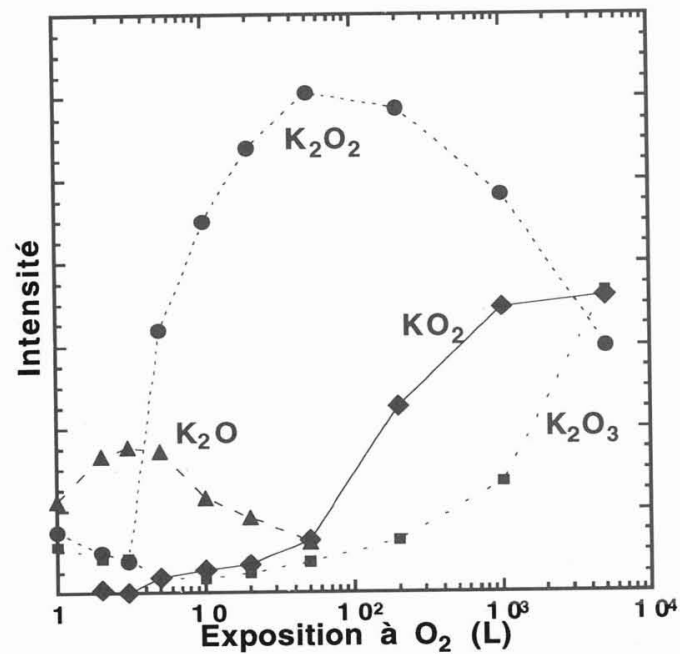

Figure 5 : Intensités XPS des oxydes de potassium en fonction de l'exposition à l'oxygène pour le cas particulier des multicouches de $\mathrm{K}$ exposées à une faible pression d'oxygène. a)

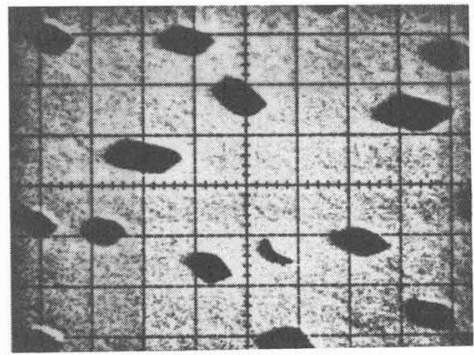

b)

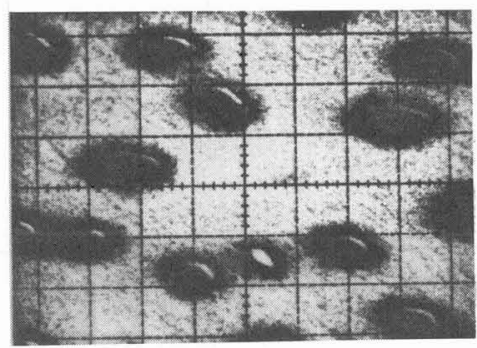

Figure 6 : Images SEM montrant les îlots de potassium s'étalant sous l'effet de la faible pression d'oxygène dans le cas des multicouches de $\mathrm{K}:$ a) $0 \mathrm{~L}$ et b) $0.2 \mathrm{~L}$ (chaque carré de la grille a une longueur horizontale de $6 \mu \mathrm{m}$ ).

Nous croyons que l'explication de ces deux mécanismes tout à fait différents réside dans le processus de formation des oxydes de potassium $\mathrm{K}_{\mathrm{x}} \mathrm{O}_{\mathrm{y}}$ qui survient lors de l'exposition. Lorsque la pression d'oxygène est faible, l'oxygène réagit faiblement avec la surface de la couche de potassium au début de l'exposition $(0-3 \mathrm{~L})$; ceci contribue à réduire l'interaction entre les atomes de potassium sans réduire leur mobilité, ce qui permet le processus observé de nivellement des îlots. Par contre, à pression élevée, l'oxygène réagit fortement avec le potassium et forme dès le début de l'exposition des oxydes à forte teneur d'oxygène qui tendent à stabiliser la couche mince à la surface des îlots et ainsi à figer ces derniers.

\subsection{Adsorption simultanée}

Dans le cas de l'adsorption simultanée, le dépôt de potassium se fait à température ambiante, en même temps que l'exposition à l'oxygène, à des pressions variées. Nos résultats [11] sont présentés à la figure 7. La légende donne la pression à laquelle l'exposition s'est effectuée, avec le rapport correspondant du nombre d'atomes d'oxygène à celui de potassium frappant la surface de silicium. Précisons que Jupille et al. [13] ont publié antérieurement des résultats en très bon accord avec ceux-ci. De plus la courbe (e) reproduit un spectre obtenu par Miranda et ses collaborateurs [7,14] avec une pression très élevée. En accord avec les résultats présentés à la figure 4 , avec une pression d'oxygène croissante, on remarque l'apparition d'oxydes de potassium impliquant une concentration plus grande d'oxygène. Pour l'exposition la plus longue (d), on 
retrouve le pic associé à l'oxyde $\mathrm{K}_{2} \mathrm{O}_{2}$ présentant une dissymétrie attribuable à la formation de $\mathrm{K}_{2} \mathrm{O}_{3}$. On constate encore une fois le lien étroit qui existe entre la pression et la nature de l'oxyde. À cause du caractère progressif du processus d'oxydation du potassium qui intervient dans le cas de l'adsorption simultanée, nous croyons que cette approche permet d'obtenir un recouvrement de $\mathrm{O}-\mathrm{K}$ homogène, contrairement à ce qui se produit dans le cas de l'adsorption séquentielle.

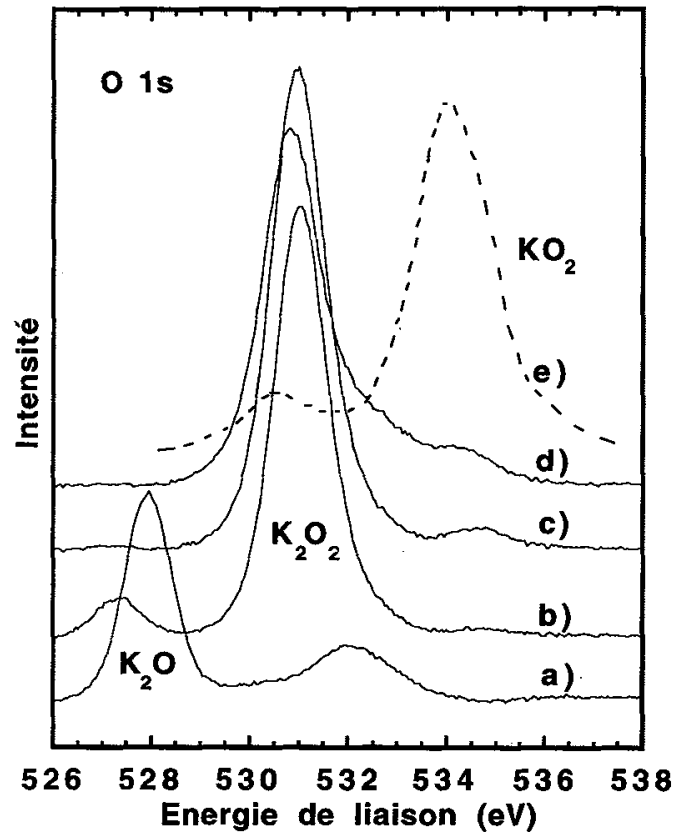

Figure 7 : Spectres XPS $\mathrm{O} 1 s$ de multicouches $\mathrm{O} / \mathrm{K}$ adsorbées sur $\mathrm{Si}(111)(300 \mathrm{~K})$ avec différentes pressions d'oxygène: (a) $2 \times 10^{-8} \mathrm{mbar}, \mathrm{O} / \mathrm{K}=0.4$; (b) $1 \times 10^{-7} \mathrm{mbar}$, $\mathrm{O} / \mathrm{K}=2$; (c) $5 \times 10^{-7} \mathrm{mbar}, \mathrm{O} / \mathrm{K}=10$; (d) $5 \times 10^{-6} \mathrm{mbar}$, $\mathrm{O} / \mathrm{K}=100$ et (e) $6.7 \times 10^{-5} \mathrm{mbar}$. Courbe (e) obtenue des travaux de Miranda et al. [7].

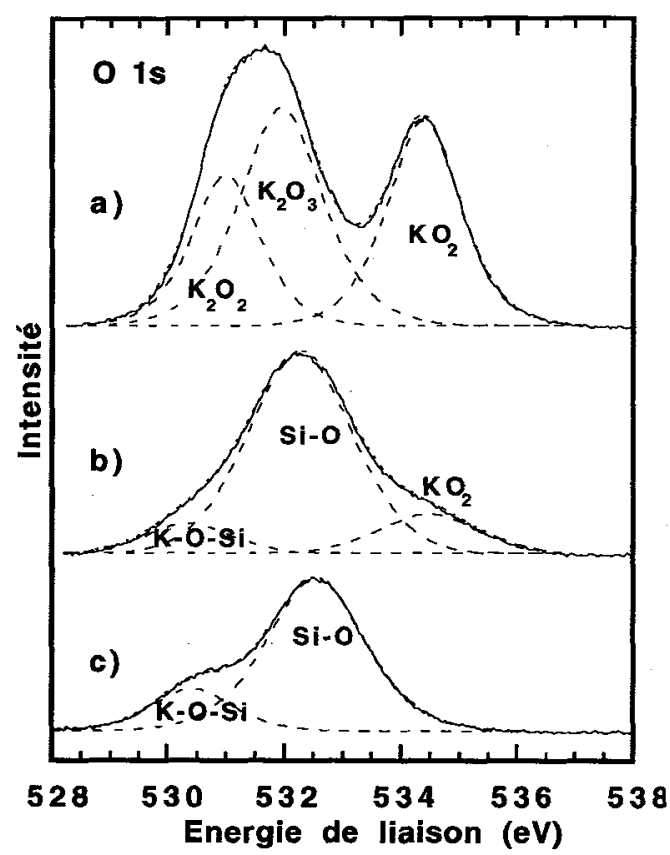

Figure 8 : Spectres XPS O $1 s$ du système $\mathrm{O} / \mathrm{K} / \mathrm{Si}(111)$ obtenus sous différentes conditions: (a) multicouches de $\mathbf{K}$ exposées à $5000 \mathrm{~L}$ d'oxygène à faible pression $\left(1 \times 10^{-8}\right.$ mbar), (b) multicouches de $\mathrm{K}$ exposées à $5000 \mathrm{~L}$ d'oxygène à haute pression ( $\left.5 \times 10^{-6} \mathrm{mbar}\right)$ et (c) monocouche de $\mathrm{K}$ exposée à $100 \mathrm{~L}$ d'oxygène.

\subsection{Cas de la monocouche}

Pour comprendre l'ensemble des divers processus qui peuvent intervenir dans ces différentes voies d'oxydation du silicium sous l'action du potassium, il faut aussi étudier le cas particulier de l'oxydation de la monocouche. Ce cas correspond à la situation de la saturation de la surface lorsque le dépôt de potassium s'effectue à température ambiante dans les meilleures conditions expérimentales de propreté. En étudiant le cas de la monocouche à basse température et à température ambiante [11], nous avons mis en évidence que ce cas diffère notablement des cas de multicouches par le type de liaison en cause, en accord avec d'autres travaux. Nous croyons qu'il n'y a pas vraiment formation d'oxydes de potassium dans ce cas, mais seulement d'une faible liaison entre l'oxygène et les atomes de silicium et de potassium (notée K-O-Si). Ceci correspond à un état intermédiaire des atomes d'oxygène qui sont ainsi placés entre la couche de potassium et le substrat de silicium, constituant ainsi une sorte d'état précurseur dans la formation des oxydes de silicium.

La figure 8 permet d'illustrer ce point. Elle montre le pic XPS O $1 s$ pour les deux cas de multicouches obtenues à $5000 \mathrm{~L}$, à basse et haute pressions respectivement, en comparaison avec le cas de la monocouche à $100 \mathrm{~L}$. On vérifie encore une fois l'important effet de la pression dans le cas des multicouches (courbes a et b), même s'il s'agit de la même couverture de potassium et de la même exposition à l'oxygène. On 
reconnaît les principaux oxydes $\mathrm{K}_{\mathrm{X}} \mathrm{O}_{\mathrm{y}}$ déjà commentés; toutefois, dàns le cas de la monocouche, il n'y a pas de nette présence d'oxydes. La déformation du pic (essentiellement associé à Si-O) est attribuée à cette faible liaison K-O-Si commentée ci-dessus. D'autre part, comme nous estimons que le processus d'oxydation dans le cas (b) vient figer les îlots de potassium, l'oxydation de la surface de ces îlots doit donc présenter une certaine analogie avec le cas (c) de la monocouche; c'est pourquoi il est logique de trouver en partie les mêmes composantes dans les deux spectres. Il va de soi que le pic principal en (b) peut aussi contenir une contribution appréciable de l'oxyde $\mathrm{K}_{2} \mathrm{O}_{3}$ à $532 \mathrm{eV}$.

Nous allons maintenant résumer nos interprétations pour les différents cas étudiés pour le système $\mathrm{O}_{2} / \mathrm{K} / \mathrm{Si}(111)$ au moyen de la représentation schématique présentée à la figure 9 . Ce schéma met de nouveau en évidence la grande influence de la morphologie de la couche de potassium sur la nature des oxydes observés. Dans le cas de la couverture d'une monocouche, le potassium agit véritablement comme un catalyseur en dissociant les molécules d'oxygène et en augmentant ainsi le nombre d'atomes disponibles pour se lier au substrat de silicium. Â plus haute couverture (cas des multicouches), la couche de potassium agit comme un réservoir d'atomes d'oxygène qui seront libérés lors d'une élévation modérée de la température. Enfin, pour obtenir une couche uniforme de $\mathrm{SiO}_{2}$, le processus d'adsorption simultanée semble être le plus prometteur, puisque les oxydes constituant le réservoir d'oxygène sont beaucoup plus homogènes.

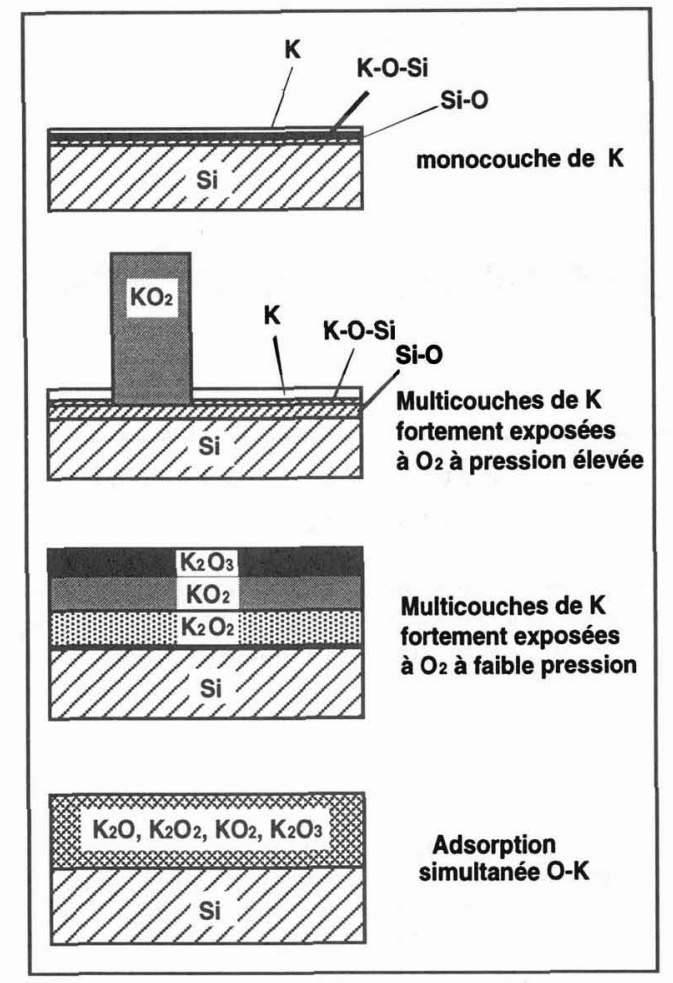

Figure 9 : Schémas représentant le système $\mathrm{O} / \mathrm{K} / \mathrm{Si}(111)$ pour différentes combinaisons de procédés d'oxydation et de recouvrements de potassium.

\section{4 Élimination du potassium}

Si l'oxydation du silicium au moyen des métaux alcalins veut se présenter comme une solution de rechange intéressante pour le procédé classique d'oxydation dans les applications technologiques, il faut faire la preuve qu'en fin de parcours toute trace de potassium est disparue de la surface résultante, sans diffusion dans le substrat. Alors que les mesures XPS permettaient toujours de confirmer l'élimination 
complète du métal alcalin après le recuit final, des mesures réalisées par SIMS par Pirug et al. [15] sont venues à un certain moment jeter des doutes sérieux sur ce point. C'est pourquoi nous avons nous-mêmes réalisé une étude SIMS des échantillons obtenus par les divers procédés discutés dans ce travail [16]. La figure 10 résume l'essentiel de nos résultats. Le diamètre du faisceau d'ions d'argon est d'environ $30 \mu \mathrm{m}$. Dans le cas des multicouches oxydées à haute pression, comme les îlots sont figés au cours du processus, du potassium reste donc emprisonné dans la couche d'oxyde résultante et le profil SIMS obtenu indique une grande quantité de potassium résiduel après le recuit final. Cependant si l'oxydation est réalisée à basse pression, le nivellement des îlots a pour effet d'éviter cet emprisonnement du potassium et ce dernier est complètement éliminé dans le recuit final. Dans ce cas, on retrouve donc un profil comparable à celui d'un échantillon de silicium propre. Ces conclusions ont également été confirmées par des mesures SIMS à haute résolution spatiale montrant que le potassium détecté dans le cas commenté était bien issu uniquement des îlots d'oxydes et qu'il n'y avait pas de diffusion de potassium dans le volume.

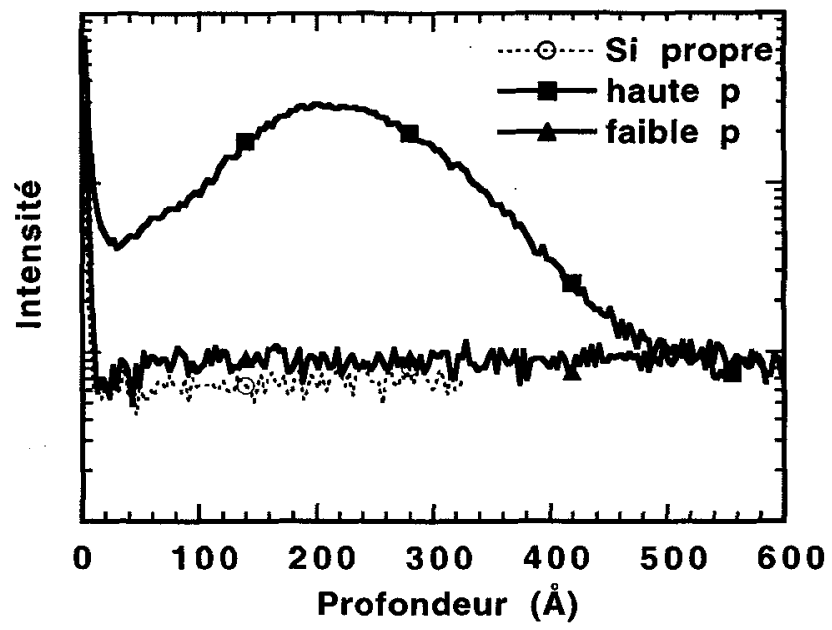

Figure 10 : Comparaison de profils SIMS (intensité du K 39) en profondeur pour différentes conditions: (i) une surface de Si(111) propre (non exposée au K); multicouches de $\mathrm{K}$ sur Si(111) refroidi à $-125^{\circ} \mathrm{C}$, ensuite exposées à $5000 \mathrm{~L}$ d'oxygène à (ii) faible pression $\left(1 \times 10^{-8} \mathrm{mbar}\right)$ ou (iii) haute pression $\left(5 \times 10^{-6} \mathrm{mbar}\right)$ subséquemment soumises à un recuit rapide $\left(\sim 700^{\circ} \mathrm{C}\right)$ afin de désorber le potassium.

\section{CONCLUSION}

Ce travail présente plusieurs aspects de l'oxydation catalytique du silicium : (i) le dépôt du potassium, (ii) l'oxydation du recouvrement de potassium, (iii) la désorption du potassium et le transfert de l'oxygène au silicium. Plusieurs conditions d'oxydation et de recouvrement en potassium ont été étudiées, ce qui a permis de dresser un tableau plus général du phénomène et d'élucider quelques controverses. Une attention particulière a été portée au cas des multicouches de potassium, permettant ainsi de mettre en évidence l'importance de la pression d'oxygène lors de l'oxydation du potassium. Le nivellement des îlots de potassium est un phénomène intéressant et très particulier, lequel suggère que d'autres études devraient être entreprises afin de mieux comprendre l'effet de l'interaction $\mathrm{O} / \mathrm{K}$ sur l'énergie de surface des îlots de potassium. La désorption thermique du potassium et le transfert de l'oxygène au substrat de silicium sont des étapes cruciales, et ce, surtout dans l'éventualité d'un transfert du procédé de la recherche à l'application. Dans ce sens, la technique SIMS a confirmé le côté prometteur de ce procédé, en particulier dans le cas de l'adsorption simultanée. 


\section{Remerciements}

Les auteurs tiennent à exprimer leur reconnaissance à plusieurs collaborateurs qui ont contribué à divers degrés au succès de ce travail : Fabrice Semond, Alain Adnot, Abdelilah Rjeb, Anthony Muscat, Daniel Guay, Patrick Soukiassian, Z.-H. Lu, I. Sproule, Luc Patry, Karl Fleury-Frenette et Luc Lévesque. Un des auteurs (B.L.) a bénéficié d'une bourse postdoctorale du Conseil de recherche en sciences naturelles et génie (CRSNG), qui subventionne également le projet avec le Fonds FCAR du Québec.

\section{Références}

[1] Ayyoob M. et Hedge M.S., Surf. Sci. 133 (1983) 516-532 .

[2] Bertel E., Netzer F.P., Rosina G. et Saalfeld H., Phys. Rev. B 39 (1989) 6082-6086 .

[3] Muscat A.J., Rjeb A. et Roy D., Surf. Sci. 302 (1994) L256-262.

[4] Pirug G., Müller O. et Bonzel H.P., dans Surface Science: Principles and Applications, vol. 73, Lamb R.N., Howe R.F. et Wandelt K. Eds. (Springer, Berlin, 1993) p.145-154 .

[5] Soukiassian P. et Starnberg H.I., dans Physics and Chemistry of Alkali Metal Adsorption, Materials Science Monograph, vol. 57, Bonzel H.P., Bradshaw A.M. et Ertl G. Eds. (Elsevier, Amsterdam, 1989) p.449-467.

[6] Riehl-Chudoba M., Nishigaki S., Huttel Y., Semond F., Brun Ph. et Soukiassian P., Appl. Surf. Sci. 65/66 (1993) 840-846.

[7] Miranda R., dans Physics and Chemistry of Alkali Metal Adsorption, Materials Science Monograph, vol. 57, Bonzel H.P., Bradshaw A.M. et Ertl G. Eds. (Elsevier, Amsterdam, 1989) p.425-447 .

[8] Michel E.G., Ortega J.E., Oellig M.C., Asensio M.C., Ferron J. et Miranda R., Phys. Rev. B 38 (1988) 13399-13406.

[9] Lamontagne B., Semond F., Adnot A., Guay D. et Roy D., Appl. Phys. A 61 (1995) 187-191.

[10] Lamontagne B., Semond F. et Roy D., J. Electron Spectrosc. Relat. Phenom. 73 (1995) 81-88.

[11] Lamontagne B., Semond F. et Roy D., Surf. Sci. 327 (1995) 371-378.

[12] Su C.Y., Lindau I., Chye P.W., Oh S.-J. et Spicer W.E., J. Electron Spectrosc. Relat. Phenom. 31 (1983) 221-259.

[13] Jupille J., Dolle P. et Besançon M., Surf. Sci. 260 (1992) 271-285 .

[14] Asensio M.C., Michel E.G., Oellig E.M. et Miranda R., Appl. Phys. Lett. 51 (1987) 1714-1716.

[15] Pirug G., Müller O. et Bonzel H.P., Appl. Surf. Sci. 70/71 (1993) 644-649 .

[16] Lamontagne B., Semond F., Adnot A. et Roy D., Appl. Surf. Sci. 90 (1995) 447-454. 